\title{
Twist-induced control of near-field heat radiation between magnetic Weyl semimetals
}

\author{
Gaomin Tang, ${ }^{1, \text { f }}$ Jun Chen, ${ }^{2}$ and Lei Zhang ${ }^{3,4, \dagger}$ \\ ${ }^{1}$ Department of Physics, University of Basel, Klingelbergstrasse 82, CH-4056 Basel, Switzerland \\ ${ }^{2}$ State Key Laboratory of Quantum Optics and Quantum Optics Devices, \\ Institute of Theoretical Physics, Shanxi University, Taiyuan 030006, China \\ ${ }^{3}$ State Key Laboratory of Quantum Optics and Quantum Optics Devices, \\ Institute of Laser Spectroscopy, Shanxi University, Taiyuan 030006, China \\ ${ }^{4}$ Collaborative Innovation Center of Extreme Optics, Shanxi University, Taiyuan 030006, China
}

\begin{abstract}
Due to the large anomalous Hall effect, magnetic Weyl semimetals can support nonreciprocal surface plasmon polariton modes in the absence of an external magnetic field. This implies that magnetic Weyl semimetals can find novel application in (thermal) photonics. In this work, we consider the near-field radiative heat transfer between two magnetic Weyl semimetal slabs and show that the heat transfer can be controlled with a relative rotation of the parallel slabs. Thanks to the intrinsic nonreciprocity of the surface modes, this so-called twisting method does not require surface structuring like periodic gratings. The twist-induced control of heat transfer is due to the mismatch of the surface modes from the two slabs with a relative rotation.
\end{abstract}

Introduction.- Near-field radiative heat transfer (NFRHT) can largely exceed the Planckian limit of black-body radiation [1] due to the contribution from surface electromagnetic modes [2-13] and attracts particular scientific interest triggered by experimental advances [14-24]. For novel applications, it is of importance to actively control NFRHT. Several strategies have been proposed, such as applying an electric field to phase-change materials [25] or ferroelectric materials [26], applying an external magnetic field to magnetooptical materials [27--32], drift currents [33, 34], and regulating the chemical potential of photons [35]. Another active control strategy is to utilize the rotational degree of freedom [36-43]. In analogy to the twistronic concept in lowdimensional materials [44-46] and photonics [47,-50], this control strategy is also called twisting method. So far, most of the proposals for the realizations of the twisting method require nanometer-sized periodic gratings to create anisotropic patterns [36, 39,-42].

Due to inherent time-reversal symmetry breaking, magnetic Weyl semimetals (WSMs), such as $\mathrm{Co}_{3} \mathrm{Sn}_{2} \mathrm{~S}_{2}$ [51, [52], $\mathrm{Ti}_{2} \mathrm{MnAl}$ [53], $\mathrm{EuCd}_{2} \mathrm{As}_{2}$ [54], $\mathrm{Co}_{2} \mathrm{MnGa}$ [55], and $\mathrm{Co}_{2} \mathrm{MnAl}$ [56], can exhibit large anomalous Hall effect so that the dielectric tensor has large off-diagonal components. This leads to the existence of nonreciprocal surface plasmon polaritons (SPPs) [57-60] and breaks the Lorentz reciprocity. The broken Lorentz reciprocity violates Kirchhoff's law of radiation and opens opportunities for a variety of radiative applications [61-67]. Compared to magneto-optical materials, magnetic WSMs break Lorentz reciprocity intrinsically in the absence of external magnetic fields and this has been studied from the perspective of (thermal) radiation very recently [6872]. Moreover, it has been shown that magnetic WSMs can exhibit nonreciprocal reflectivity without surface structuring using a planar interface [70].

In this Letter, we employ the intrinsic nonreciprocity of the surface modes in magnetic WSMs and demonstrate that NFRHT between magnetic WSMs can be actively controlled via twist. We will first show how the nonreciprocal dispersion of SPPs changes with the incidence plane of the light. Using fluctuational electrodynamics, we will study the implications of nonreciprocity on NFRHT and the twisting effects between two WSM slabs.

Surface plasmon polaritons.- In WSM, either inversion or time-reversal symmetry needs to be broken to split a doubly degenerate Dirac point into a pair of Weyl nodes with opposite chirality [73, 74]. Each pair of Weyl nodes are separated in momentum space (denoted by wave vector $2 \mathbf{b}$ ) by breaking time-reversal symmetry or with an energy of $2 \hbar b_{0}$ by breaking inversion symmetry. The presence of Weyl nodes changes the electromagnetic response and the displacement electric field for WSM in the frequency domain is written as [75]

$$
\mathbf{D}=\epsilon_{0} \epsilon_{d} \mathbf{E}+\frac{i e^{2}}{4 \pi^{2} \hbar \omega}\left(-2 b_{0} \mathbf{B}+2 \mathbf{b} \times \mathbf{E}\right)
$$

with $\omega$ the angular frequency. The dielectric function $\epsilon_{d}$ is expressed as $\epsilon_{d}=\epsilon_{b}+i \sigma / \omega$ where $\epsilon_{b}$ is the background permittivity and $\sigma$ the bulk conductivity. It is seen from Eq. (1) that $b_{0}$ gives rise to the chiral magnetic effect and $\mathbf{b}$ the anomalous Hall effect. This implies that magnetic WSMs with broken time-reversal symmetry can give rise to the anomalous Hall effect. Considering $2 \mathbf{b}$ along the $y$-direction in momentum space $\left(\mathbf{b}=b \hat{q}_{y}\right)$ and inversion symmetric system with $b_{0}=0$, we have $\mathbf{D}=\epsilon_{0} \overline{\bar{\epsilon}} \mathbf{E}$ in the Cartesian coordinate system where the dielectric tensor is

$$
\overline{\bar{\epsilon}}(\omega)=\left[\begin{array}{ccc}
\epsilon_{d} & 0 & i \epsilon_{a} \\
0 & \epsilon_{d} & 0 \\
-i \epsilon_{a} & 0 & \epsilon_{d}
\end{array}\right]
$$

with $\epsilon_{a}=b e^{2} /\left(2 \pi^{2} \epsilon_{0} \hbar \omega\right)$. It has been reported that $\epsilon_{a}$ can be comparable to $\epsilon_{d}$ in the infrared region which is of most interest for thermal applications [68-71].

We first discuss the dispersion relations of SPPs at the planar interface between WSM and air by considering only one WSM slab. With the incidence plane at azimuthal angle $\phi$ with respect to the $x$-axis, which is the $x^{\prime}-z$ plane shown in 
Figure 1(d), the dielectric tensor is transformed to

$$
\overline{\bar{\epsilon}}^{\prime}(\omega)=\mathcal{R} \overline{\bar{\epsilon}}(\omega) \mathcal{R}^{T}=\left[\begin{array}{ccc}
\epsilon_{d} & 0 & i \epsilon_{a} \cos \phi \\
0 & \epsilon_{d} & i \epsilon_{a} \sin \phi \\
-i \epsilon_{a} \cos \phi & -i \epsilon_{a} \sin \phi & \epsilon_{d}
\end{array}\right]
$$

where $\mathcal{R}$ is the rotation matrix of angle $\phi$. We start from Maxwell curl equations

$$
\nabla \times \mathbf{E}=-\partial_{t} \mathbf{B}, \quad \nabla \times \mathbf{H}=\partial_{t} \mathbf{D},
$$

with $\mathbf{B}=\mu_{0} \mu \mathbf{H}$ and $\mathbf{D}=\epsilon_{0} \overline{\bar{\epsilon}}^{\prime} \mathbf{E}$. Since the SPP is transverse magnetic (or $p$-polarized) mode, the magnetic fields in air $\left(\mathbf{H}_{0}\right)$ and in WSM $\left(\mathbf{H}_{1}\right)$ are written in the forms as

$$
\begin{array}{ll}
\mathbf{H}_{0}\left(x^{\prime}, z, t\right)=\hat{y}^{\prime} H e^{i q x^{\prime}-i \beta_{0} z} e^{-i \omega t}, & \operatorname{Im}\left(\beta_{0}\right)<0, \\
\mathbf{H}_{1}\left(x^{\prime}, z, t\right)=\hat{y}^{\prime} H e^{i q x^{\prime}+i \beta_{1} z} e^{-i \omega t}, & \operatorname{Im}\left(\beta_{1}\right)<0,
\end{array}
$$

where $q$ is the in-plane wave vector. The out-of-plane wave vectors in air and WSM are denoted as $\beta_{0}$ and $\beta_{1}$, respectively. Using Maxwell equations in the WSM and air, respectively, one has

$$
\beta_{0}^{2}+q^{2}=k_{0}^{2}, \quad \beta_{1}^{2}+q^{2}=\mu \epsilon_{\mathrm{eff}} k_{0}^{2},
$$

with $k_{0}=\omega / c$ the wave vector in air and the dielectric function $\epsilon_{\text {eff }}=\epsilon_{d}-\left(\cos \phi \epsilon_{a}\right)^{2} / \epsilon_{d}$. Using the interface condition of electric field, the implicit dispersion relation for the SPP is obtained as

$$
\epsilon_{\mathrm{eff}} \beta_{0}+\beta_{1}+i \cos \phi \epsilon_{a} q / \epsilon_{d}=0 .
$$

It can be seen from Eq. 8 that the dispersion is nonreciprocal as long as $\cos \phi \neq 0$ and is reciprocal in the Faraday configurations with $\phi=\pi / 2$ or $\phi=3 \pi / 2$. From Eqs. (7) and (8), the dispersion relation of SPP can be numerically obtained. The bulk plasmon dispersion is found as $q= \pm \sqrt{\mu \epsilon_{\mathrm{eff}}} k_{0}$ with $\epsilon_{\text {eff }}>0$. We consider the case with the relative permeability $\mu$ to be 1 .

The bulk conductivity $\sigma$ can be obtained using the KuboGreenwood formalism to a two-band model with spin degeneracy as [58, 76]

$$
\begin{array}{r}
\sigma=\frac{g r_{s}}{6} \Omega G\left(\frac{\hbar \Omega}{2}\right)+i \frac{g r_{s}}{6 \pi}\left\{\frac{4}{\hbar^{2} \Omega}\left[E_{F}^{2}+\frac{\pi^{2}}{3}\left(k_{B} T\right)^{2}\right]\right. \\
\left.+8 \Omega \int_{0}^{E_{c}} \frac{G(E)-G(\hbar \Omega / 2)}{(\hbar \Omega)^{2}-4 E^{2}} E d E\right\} .
\end{array}
$$

Here, $g$ is the number of Weyl nodes, $r_{s}=e^{2} /\left(4 \pi \epsilon_{0} \hbar v_{F}\right)$ is the effective fine-structure constant with Fermi velocity $v_{F}, \Omega=\omega+i 2 \pi \tau^{-1}$ with the Drude damping rate $\tau^{-1}$, $G(E)=n(-E)-n(E)$ with the Fermi distribution function $n(E), E_{F}$ is the chemical potential, and $E_{c}$ is the cutoff energy. Following Refs. [68-71], we take the parameters $b=2 \times 10^{9} \mathrm{~m}^{-1}, \epsilon_{b}=6.2, g=2, v_{F}=0.83 \times 10^{5} \mathrm{~m} / \mathrm{s}$, $\tau=1000 \mathrm{fs}, E_{F}=0.15 \mathrm{eV}$ at temperature $T=300 \mathrm{~K}$, and $E_{c}=3 E_{F}$. The parameters are close to the reported values for $\mathrm{Co}_{3} \mathrm{Sn}_{2} \mathrm{~S}_{2}$ [51, 52] and the room temperature WSM $\mathrm{Co}_{2} \mathrm{MnGa}$ [55].
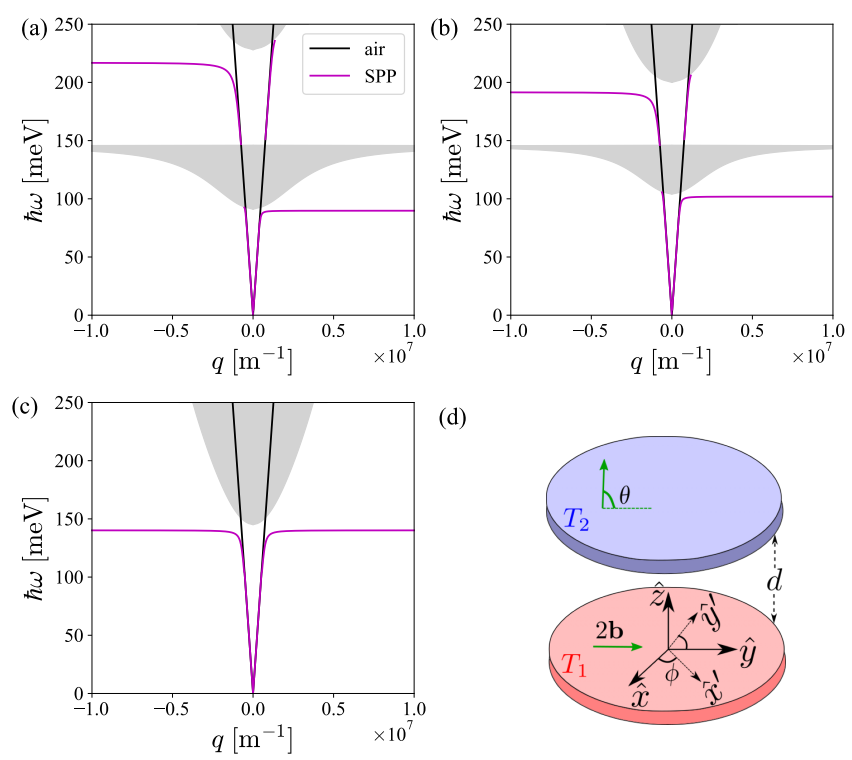

(d)

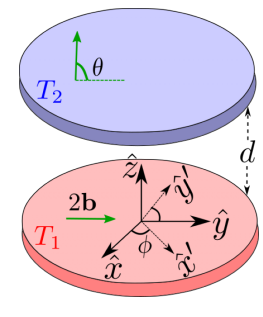

FIG. 1. The dispersion of surface plasmon polaritons (magenta lines) with different azimuthal angles of incidence: (a) $\phi=0$, (b) $\phi=$ $\pi / 4$, and (c) $\phi=\pi / 2$. The black lines are the linear dispersion relation in air (or vacuum). The gray regions show the continua of the bulk plasmon modes in Weyl semimetal. (d) Schematic setup for near-field heat radiation between two Weyl semimetals with gap separation $d$ and twist angle $\theta$. The twist angle is defined as the angle between the Weyl node separations in the bottom and top Weyl semimetals.

Figures. 1] a)-11.c) show the dispersions of SPPs at different incidence planes characterized by the azimuthal angle $\phi$. The gray regions show the continua of bulk plasmon modes which are reciprocal. At $\phi=0$ (Voigt configuration), the nonreciprocity of the SPPs is clearly identified by the asymmetry with respect to the wave vector $q$. There are two continua of bulk plasmon modes: one is lower in frequency and the other higher. The low-frequency continuum separates the SPPs into two branches. With increasing the azimuthal angle from $\phi=0$ to $\phi=\pi / 2$ (Faraday configuration), the lowfrequency continuum shrinks and the degrees of nonreciprocity decreases. At $\phi=\pi / 2$, the low-frequency continuum vanishes and the SPP dispersion becomes strictly reciprocal.

Here, we only show the dispersions of SPPs which are $p$ polarized. There exist $s$-polarized surface modes as well. As it was shown in Ref. [71], the $s$-polarized modes are nonreciprocal between Voigt and Faraday configurations. Since it is the $p$-polarized modes that dominate the NFRHT in WSM, the twist-induced near-field thermal control is mainly due to the nonreciprocity of SPPs.

Near-field radiative heat transfer-- We now consider the NFRHT between two magnetic WSM slabs of the same properties with temperatures $T_{1(2)}=T \pm \Delta T / 2$. The two slabs are placed in parallel and separated by an air gap with distance $d$ [See Figure 11(d)]. The twist angle $\theta$ is the angle between the Weyl node separations in the two slabs and can be changed by rotating one of the WSMs. From the fluctuational electrody- 

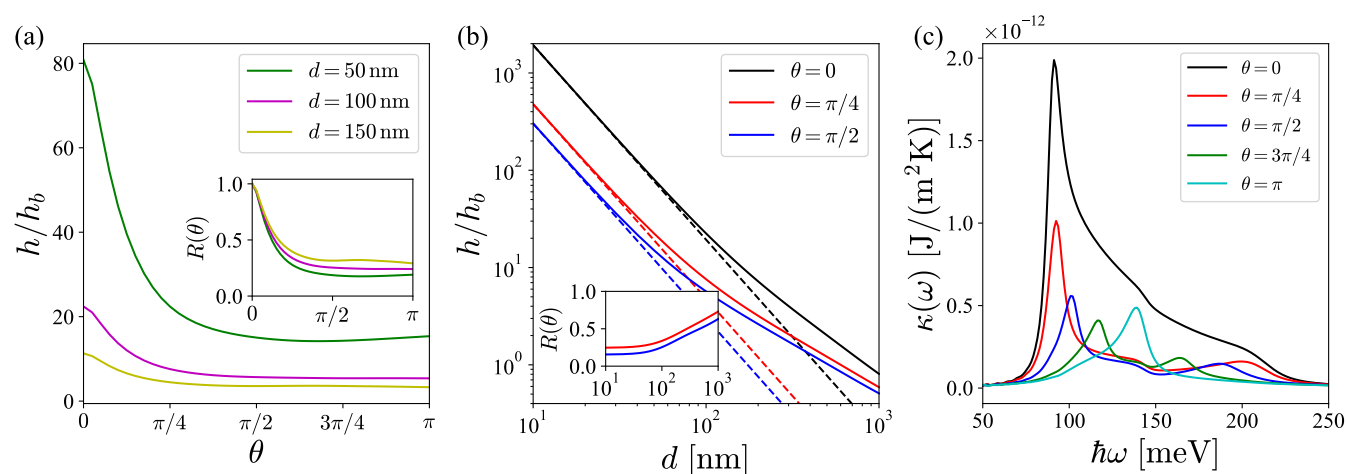

FIG. 2. (a) Scaled heat transfer coefficient $h / h_{b}$ versus twist angle $\theta$ at different gap separations $d$. (b) Scaled heat transfer coefficient $h / h_{b}$ versus gap separation $d$ under different twist angles. The corresponding dashed lines are plotted using $h \propto d^{-2}$. The thermal switch ratios $R(\theta)$ are shown as inset in (a) and (b). (c) Spectral function $\kappa(\omega)$ at different twist angles $\theta$ with $d=100 \mathrm{~nm}$.

namics [3, 12], the radiative heat transfer coefficient (HTC) $h(\theta)$ at temperature $T$ is given by

$$
h(\theta)=\int_{0}^{\infty} \frac{d \omega}{2 \pi} \hbar \omega N^{\prime} \int_{0}^{\infty} \frac{d q}{2 \pi} q \int_{0}^{2 \pi} \frac{d \phi}{2 \pi} \xi(\omega, q, \phi),
$$

where $q$ is the in-plane wave vector and $N^{\prime}$ is the derivative of Bose-Einstein distribution $N=1 /\left[e^{\hbar \omega /\left(k_{B} T\right)}-1\right]$ with respect to the temperature and is expressed as

$$
N^{\prime} \equiv \partial N / \partial T=\frac{\hbar \omega e^{\hbar \omega /\left(k_{B} T\right)}}{k_{B} T^{2}\left[e^{\hbar \omega /\left(k_{B} T\right)}-1\right]^{2}} .
$$

The photonic transmission coefficient $\xi(\omega, q, \phi)$ is expressed as

$$
\xi= \begin{cases}\operatorname{Tr}\left[\left(\mathbf{I}-\mathbf{R}_{2}^{\dagger} \mathbf{R}_{2}\right) \mathbf{D}\left(\mathbf{I}-\mathbf{R}_{1} \mathbf{R}_{1}^{\dagger}\right) \mathbf{D}^{\dagger}\right], & q<k_{0} \\ \operatorname{Tr}\left[\left(\mathbf{R}_{2}^{\dagger}-\mathbf{R}_{2}\right) \mathbf{D}\left(\mathbf{R}_{1}-\mathbf{R}_{1}^{\dagger}\right) \mathbf{D}^{\dagger}\right] e^{-2\left|\beta_{0}\right| d}, & q>k_{0}\end{cases}
$$

The identity matrix is denoted as $\mathbf{I}$. The reflection coefficient matrix $\mathbf{R}_{n}$ at the interface between air and WSM $n$ with $n=$ 1,2 has the form

$$
\mathbf{R}_{n}=\left[\begin{array}{ll}
r_{n}^{p p} & r_{n}^{p s} \\
r_{n}^{s p} & r_{n}^{s s}
\end{array}\right]
$$

and is provided in the Supporting Information. Furthermore, $\mathbf{D}=\left(\mathbf{I}-\mathbf{R}_{1} \mathbf{R}_{2} e^{-2 i \beta_{0} d}\right)^{-1}$ is the Fabry-Perot-like denominator matrix. The near- and far-field regimes are defined by the conditions $q>k_{0}$ and $q<k_{0}$, respectively. Here, we consider the situation of $T=300 \mathrm{~K}$, which can be achieved using room temperature WSMs discovered recently, such as $\mathrm{Co}_{2} \mathrm{MnGa}[55]$ and $\mathrm{Co}_{2} \mathrm{MnAl}$ [56]. We consider the HTC to be scaled by the corresponding black-body limit $h_{b}=4 \sigma_{\mathrm{SB}} T^{3}$ with the Stefan-Boltzmann constant $\sigma_{\mathrm{SB}}=$ $\pi^{2} k_{B}^{4} /\left(60 \hbar^{3} c^{2}\right)$. One can calculate that $h_{b}$ is $6.12 \mathrm{~W} / \mathrm{m}^{2} \mathrm{~K}$ under $T=300 \mathrm{~K}$.

In Figure 2(a), we show the scaled HTC $h(\theta) / h_{b}$ versus the twist angle $\theta$ at different gap distances $d$. Since $h(\theta)$ is symmetric with respect to $\theta=\pi$, only the part of $\theta \in[0, \pi]$ is shown. The HTC is maximal at $\theta=0$, decreases with increasing $\theta$ to $\theta=\pi / 2$ and remains almost unchanged for $\pi / 2 \leq \theta \leq \pi$. The corresponding thermal switch ratios, which are defined as $R(\theta)=h(\theta) / h(\theta=0)$, are shown as an inset. Compared to the HTC, the thermal switch ratio is less sensitive to the gap distance. The tunability reported here can be comparable to those by gratings [36, 40, 42] and by rotating a magnetic field in the case of magneto-optical materials [29]. Figure 2 b) shows the dependence of HTC on gap separation $d$. The heat transfer diverges as $d^{-2}$ at very small distances as shown in dashed lines which was predicted by Loomis and Maris [4].

The spectral function $\kappa(\omega)$ of HTC is defined through $h=\int_{0}^{\infty} \kappa(\omega) d \omega$ and its behaviors for different twist angles $\theta$ are shown in Figure 2(c). We first focus on the parallel case $(\theta=0)$, of which the photonic transmission coefficients $\xi(\omega, q, \phi)$ against $\hbar \omega$ and $q$ for different $\phi$ in Figures 3 a)3 (c) with $d=100 \mathrm{~nm}$. Close to or in the far-field regions, $\xi(\omega, q, \phi)$ are less than or equal to 2 , which is due to the contributions from both $p$ - and $s$-polarized modes. The contributions to $\xi$ in near field are dominated by the SPPs which are $p$-polarized. This is confirmed by Figure $\mathrm{S} 1$ in the Supporting Information where the contribution from the $p$-polarized mode $\left[r_{n}^{p p}\right.$ in Eq. [42)] on the spectral function is very close to that from all modes. For $\theta=0$, the individual SPP from the two WSMs are identical so that they couple with each other for the whole range of $\phi$ with $\phi \in[0,2 \pi]$. This explains that HTC is maximal at $\theta=0$. The near-field regions where $\xi$ are close to 1 are consistent with the odd (dashed lines) and even (dash-dotted lines) SPP modes, which are given by

$$
\begin{aligned}
\epsilon_{\mathrm{eff}} \beta_{0}+\operatorname{coth}\left(\left|\beta_{0}\right| d / 2\right)\left(\beta_{1}+i \cos \phi \epsilon_{a} q / \epsilon_{d}\right) & =0, \\
\epsilon_{\mathrm{eff}} \beta_{0}+\tanh \left(\left|\beta_{0}\right| d / 2\right)\left(\beta_{1}+i \cos \phi \epsilon_{a} q / \epsilon_{d}\right) & =0,
\end{aligned}
$$

respectively. Similarly to Figures 1 (a) -1 (c), the degrees of nonreciprocity for both the odd and even modes decrease from $\phi=0$ to $\phi=\pi / 2$ at which the modes become reciprocal. Due to the nonreciprocity of the SPPs, the resonant frequency ranges are different for different $\phi$ with $0 \leq \phi \leq \pi$ at a 

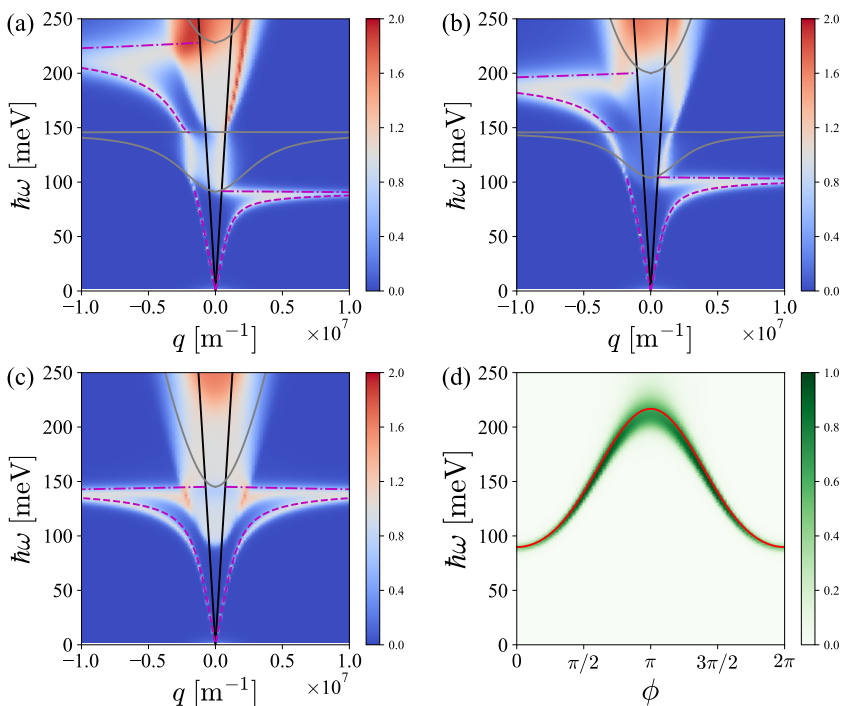

FIG. 3. The photonic transmission coefficients $\xi(\omega, q, \phi)$ are plotted against $\hbar \omega$ and $q$ for different azimuthal angles of incidence without twisting $(\theta=0)$ : (a) $\phi=0$, (b) $\phi=\pi / 4$, and (c) $\phi=\pi / 2$. The black lines depict the linear dispersion in air (or vacuum). The gray lines mark the continuum regions of the bulk plasmon modes as in Figures 1 a)-1 (c). The dashed and dash-dotted magenta lines indicate the odd and even surface plasmon polariton modes, respectively. (d) $\xi(\omega, q, \phi)$ plotted against $\hbar \omega$ and $\phi$ at twist angle $\theta=0$ and $q=$ $10^{7} \mathrm{~m}^{-1}$. The red line is obtained using Eq. 8). The gap separation is $d=100 \mathrm{~nm}$.

given wave vector $q$. This can be seen from Figure 3 d) where $\xi(\omega, q, \phi)$ is shown against $\hbar \omega$ and $\phi$ at $q=10^{7} \mathrm{~m}^{-1}$ under $d=100 \mathrm{~nm}$. Because of the large nonreciprocity of the SPPs, the whole resonant frequency range is very broad (from about $90 \mathrm{meV}$ at $\phi=0$ to about $220 \mathrm{meV}$ at $\phi=\pi$ ). This can be seen from the spectral function at $\theta=0$ shown in Figure 2(c) as well.

Now we analyze the twisting effects. In Figure 4, the photonic transmission coefficients $\xi(\omega, q, \phi)$ are plotted against $\hbar \omega$ and $\phi$ for different twist angles at $q=10^{7} \mathrm{~m}^{-1}$. The red lines are plotted using the SPP dispersion relation, Eq. (8), and the blues lines are obtained by performing the shift $\phi \rightarrow \phi+\theta$. Due to the twist, the red and blue lines cross at two points. The surface modes from each interface can only couple around the two crossing points in the $\omega-\phi$ space and this results in two resonant regions with $\xi$ being close to 1 [See Figure 4]. The resonant regions correspond to the resonant peaks in the spectral function shown in Figure 2(c). Due to the mismatch of the surface modes from the two interfaces, the spectral function is reduced and so is the HTC.

To conclude, we have considered the situation where the near-field radiative heat transfer between two magnetic Weyl semimetals are dominated by the nonreciprocal surface plasmon polaritons. Due to the intrinsic nonreciprocity, the heat transfer can be effectively controlled by a relative rotaion of parallel slabs (or twist) without surface structuring or external field.
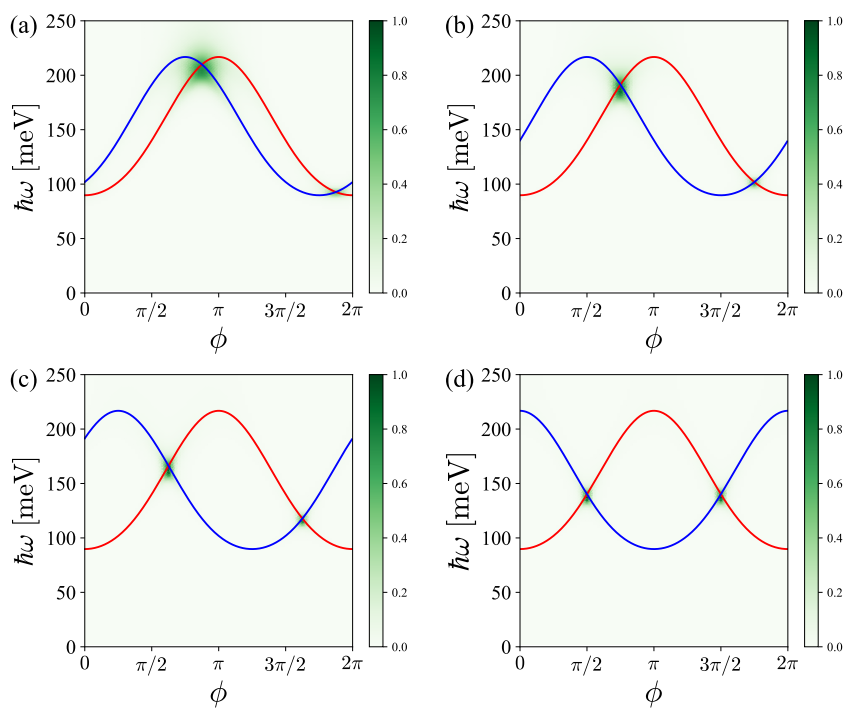

FIG. 4. The photonic transmission coefficients $\xi(\omega, q, \phi)$ are plotted against $\hbar \omega$ and $\phi$ at different twist angles with (a) $\theta=\pi / 4$, (b) $\theta=\pi / 2$, (c) $\theta=3 \pi / 4$, and (d) $\theta=\pi$ at $q=1 / d=10^{7} \mathrm{~m}^{-1}$ with $d=100 \mathrm{~nm}$. The red lines are the same as the one in Figure 3.d). The blue lines are obtained by shifting the red lines using $\phi \rightarrow \phi+\theta$.

Acknowledgments.- G.T. thanks Christoph Bruder for discussions and acknowledges financial support from the Swiss National Science Foundation (SNSF) and the NCCR Quantum Science and Technology. J.C. and L.Z. acknowledges the support from the National Natural Science Foundation of China (Grants No. 12074230, 12047571), National Key R\&D Program of China under Grants No. 2017YFA0304203, 1331KSC, Shanxi Province 100-Plan Talent Program.

* gaomin.tang@unibas.ch

† zhanglei@sxu.edu.cn

[1] M. Planck and M. Masius, The Theory of Heat Radiation (P. Blakiston's Son \& Co, New York, 1914).

[2] C. Hargreaves, Anomalous radiative transfer between closelyspaced bodies, Phys. Lett. A 30, 491 (1969)

[3] D. Polder and M. Van Hove, Theory of radiative heat transfer between closely spaced bodies, Phys. Rev. B 4, 3303 (1971)

[4] J. J. Loomis and H. J. Maris, Theory of heat transfer by evanescent electromagnetic waves, Phys. Rev. B 50, 18517 (1994)

[5] J. Xu, K. Läuger, R. Möller, K. Dransfeld, and I. H. Wilson, Heat transfer between two metallic surfaces at small distances, J. Appl. Phys. 76, 7209 (1994)

[6] K. Joulain, J.-P. Mulet, F. Marquier, R. Carminati, and J.-J. Greffet, Surface electromagnetic waves thermally excited: Radiative heat transfer, coherence properties and Casimir forces revisited in the near field, Surface Science Reports 57, 59 (2005)

[7] A. I. Volokitin and B. N. J. Persson, Near-field radiative heat transfer and noncontact friction, Rev. Mod. Phys. 79, 1291 (2007)

[8] B. Song, A. Fiorino, E. Meyhofer, and P. Reddy, Near-field radiative thermal transport: From theory to experiment, AIP Adv. 


\section{$\mathbf{5 ,} 053503(2015)$}

[9] X. Liu, L. Wang, and Z. M. Zhang, Near-field thermal radiation: Recent progress and outlook, Nanoscale and Microscale Thermophys. Eng. 19, 98 (2015)

[10] J. C. Cuevas and F. J. García-Vidal, Radiative heat transfer, ACS Photonics 5, 3896 (2018)

[11] G. Tang, H. H. Yap, J. Ren, and J.-S. Wang, Anomalous nearfield heat transfer in carbon-based nanostructures with edge states, Phys. Rev. Applied 11, 031004 (2019)

[12] Z. M. Zhang, Nano/Microscale Heat Transfer (Springer, Cham, 2020).

[13] S.-A. Biehs, R. Messina, P. S. Venkataram, A. W. Rodriguez, J. C. Cuevas, and P. Ben-Abdallah, Near-field radiative heat transfer in many-body systems (2020), arXiv:2007.05604

[14] R. St-Gelais, B. Guha, L. Zhu, S. Fan, and M. Lipson, Demonstration of strong near-field radiative heat transfer between integrated nanostructures, Nano Letters 14, 6971 (2014).

[15] B. Song, Y. Ganjeh, S. Sadat, D. Thompson, A. Fiorino, V. Fernández-Hurtado, J. Feist, F. J. Garcia-Vidal, J. C. Cuevas, P. Reddy, and E. Meyhofer, Enhancement of near-field radiative heat transfer using polar dielectric thin films, Nature Nanotechnology 10, 253 (2015)

[16] K. Kim, B. Song, V. Fernández-Hurtado, W. Lee, W. Jeong, L. Cui, D. Thompson, J. Feist, M. T. H. Reid, F. J. GarcíaVidal, J. C. Cuevas, E. Meyhofer, and P. Reddy, Radiative heat transfer in the extreme near field, Nature 528, 387 (2015).

[17] B. Song, D. Thompson, A. Fiorino, Y. Ganjeh, P. Reddy, and E. Meyhofer, Radiative heat conductances between dielectric and metallic parallel plates with nanoscale gaps, Nature Nanotechnology 11, 509 (2016).

[18] M. P. Bernardi, D. Milovich, and M. Francoeur, Radiative heat transfer exceeding the blackbody limit between macroscale planar surfaces separated by a nanosize vacuum gap, Nature Communications 7, 12900 (2016)

[19] L. Cui, W. Jeong, V. Fernández-Hurtado, J. Feist, F. J. GarcíaVidal, J. C. Cuevas, E. Meyhofer, and P. Reddy, Study of radiative heat transfer in ångström- and nanometre-sized gaps, Nat. Commun. 8, 14479 (2017)

[20] K. Kloppstech, N. Könne, S.-A. Biehs, A. W. Rodriguez, L. Worbes, D. Hellmann, and A. Kittel, Giant heat transfer in the crossover regime between conduction and radiation, Nature Communications 8, 14475 (2017)

[21] M. Ghashami, H. Geng, T. Kim, N. Iacopino, S. K. Cho, and K. Park, Precision measurement of phonon-polaritonic near-field energy transfer between macroscale planar structures under large thermal gradients, Phys. Rev. Lett. 120, 175901 (2018)

[22] A. Fiorino, D. Thompson, L. Zhu, B. Song, P. Reddy, and E. Meyhofer, Giant enhancement in radiative heat transfer in sub-30 nm gaps of plane parallel surfaces, Nano Letters 18, 3711 (2018)

[23] J. DeSutter, L. Tang, and M. Francoeur, A near-field radiative heat transfer device, Nature Nanotechnology 14, 751 (2019).

[24] L. Tang, J. DeSutter, and M. Francoeur, Near-field radiative heat transfer between dissimilar materials mediated by coupled surface phonon- and plasmon-polaritons, ACS Photonics 7, 1304 (2020)

[25] P. J. van Zwol, K. Joulain, P. Ben Abdallah, J. J. Greffet, and J. Chevrier, Fast nanoscale heat-flux modulation with phasechange materials, Phys. Rev. B 83, 201404 (2011).

[26] Y. Huang, S. V. Boriskina, and G. Chen, Electrically tunable near-field radiative heat transfer via ferroelectric materials, Applied Physics Letters 105, 244102 (2014)

[27] E. Moncada-Villa, V. Fernández-Hurtado, F. J. García-Vidal,
A. García-Martín, and J. C. Cuevas, Magnetic field control of near-field radiative heat transfer and the realization of highly tunable hyperbolic thermal emitters, Phys. Rev. B 92, 125418 (2015)

[28] I. Latella and P. Ben-Abdallah, Giant thermal magnetoresistance in plasmonic structures, Phys. Rev. Lett. 118, 173902 (2017)

[29] R. M. Abraham Ekeroth, P. Ben-Abdallah, J. C. Cuevas, and A. García-Martín, Anisotropic thermal magnetoresistance for an active control of radiative heat transfer, ACS Photonics 5, 705 (2018)

[30] H. Wu, Y. Huang, L. Cui, and K. Zhu, Active magneto-optical control of near-field radiative heat transfer between graphene sheets, Phys. Rev. Applied 11, 054020 (2019)

[31] E. Moncada-Villa and J. C. Cuevas, Magnetic field effects in the near-field radiative heat transfer between planar structures, Phys. Rev. B 101, 085411 (2020)

[32] E. Moncada-Villa and J. C. Cuevas, Near-field radiative heat transfer between one-dimensional magneto-photonic crystals (2020), arXiv:2010.02794

[33] J. Peng and J.-S. Wang, Current-induced heat transfer in double-layer graphene, arXiv:1805.09493

[34] Y. Zhang, C.-L. Zhou, L. Qu, and H.-L. Yi, Active control of near-field radiative heat transfer through nonreciprocal graphene surface plasmons, Applied Physics Letters 116, $151101(2020)$

[35] K. Chen, P. Santhanam, S. Sandhu, L. Zhu, and S. Fan, Heatflux control and solid-state cooling by regulating chemical potential of photons in near-field electromagnetic heat transfer, Phys. Rev. B 91, 134301 (2015)

[36] S.-A. Biehs, F. S. S. Rosa, and P. Ben-Abdallah, Modulation of near-field heat transfer between two gratings, Applied Physics Letters 98, 243102 (2011)

[37] X. Liu, J. Shen, and Y. Xuan, Pattern-free thermal modulator via thermal radiation between van der Waals materials, Journal of Quantitative Spectroscopy and Radiative Transfer 200, 100 (2017)

[38] Y. Zhang, H.-L. Yi, and H.-P. Tan, Near-field radiative heat transfer between black phosphorus sheets via anisotropic surface plasmon polaritons, ACS Photonics 5, 3739 (2018)

[39] M. He, H. Qi, Y. Ren, Y. Zhao, and M. Antezza, Active control of near-field radiative heat transfer by a graphene-gratings coating-twisting method, Opt. Lett. 45, 2914 (2020).

[40] M.-J. He, H. Qi, Y.-T. Ren, Y.-J. Zhao, Y. Zhang, J.-D. Shen, and M. Antezza, Radiative thermal switch driven by anisotropic black phosphorus plasmons, Opt. Express 28, 26922 (2020)

[41] M. Luo, J. Zhao, and M. Antezza, Near-field radiative heat transfer between twisted nanoparticle gratings, Applied Physics Letters 117, 053901 (2020)

[42] C. Zhou, X. Wu, Y. Zhang, H. Yi, and M. Antezza, Polariton topological transition effects on radiative heat transfer (2020), arXiv:2011.02263.

[43] J. Peng, G. Tang, L. Wang, R. Macêdo, H. Chen, and J. Ren, Twist-induced near-field thermal switch using nonreciprocal surface magnon-polaritons (2020), arXiv:2012.14733.

[44] Y. Cao, V. Fatemi, S. Fang, K. Watanabe, T. Taniguchi, E. Kaxiras, and P. Jarillo-Herrero, Unconventional superconductivity in magic-angle graphene superlattices, Nature 556, 43 (2018)

[45] Y. Cao, D. Chowdhury, D. Rodan-Legrain, O. Rubies-Bigorda, K. Watanabe, T. Taniguchi, T. Senthil, and P. Jarillo-Herrero, Strange metal in magic-angle graphene with near Planckian dissipation, Phys. Rev. Lett. 124, 076801 (2020)

[46] W. Chen, Z. Sun, Z. Wang, L. Gu, X. Xu, S. Wu, and C. Gao, Direct observation of van der Waals stacking-dependent inter- 
layer magnetism, Science 366, 983 (2019)

[47] P. Wang, Y. Zheng, X. Chen, C. Huang, Y. V. Kartashov, L. Torner, V. V. Konotop, and F. Ye, Localization and delocalization of light in photonic moiré lattices, Nature 577, 42 (2020)

[48] Q. Fu, P. Wang, C. Huang, Y. V. Kartashov, L. Torner, V. V. Konotop, and F. Ye, Optical soliton formation controlled by angle twisting in photonic moiré lattices, Nature Photonics 14, $663(2020)$

[49] G. Hu, Q. Ou, G. Si, Y. Wu, and A. Alú, Topological polaritons and photonic magic angles in twisted $\alpha-\mathrm{MoO}_{3}$ bilayers, Nature 582, 209 (2020)

[50] G. Hu, A. Krasnok, Y. Mazor, C. W. Qiu, and A. Alú, Moiré hyperbolic metasurfaces, Nano Letters 20, 3217 (2020)

[51] Q. Wang, Y. Xu, R. Lou, Z. Liu, M. Li, Y. Huang, D. Shen, $\mathrm{H}$. Weng, S. Wang, and H. Lei, Large intrinsic anomalous Hall effect in half-metallic ferromagnet $\mathrm{Co}_{3} \mathrm{Sn}_{2} \mathrm{~S}_{2}$ with magnetic Weyl fermions, Nature Communications 9, 3681 (2018)

[52] E. Liu, Y. Sun, N. Kumar, L. Muechler, A. Sun, L. Jiao, S.-Y. Yang, D. Liu, A. Liang, Q. Xu, J. Kroder, V. Süß, H. Borrmann, C. Shekhar, Z. Wang, C. Xi, W. Wang, W. Schnelle, S. Wirth, Y. Chen, S. T. B. Goennenwein, and C. Felser, Giant anomalous Hall effect in a ferromagnetic kagome-lattice semimetal, Nature Physics 14, 1125 (2018)

[53] W. Shi, L. Muechler, K. Manna, Y. Zhang, K. Koepernik, R. Car, J. van den Brink, C. Felser, and Y. Sun, Prediction of a magnetic Weyl semimetal without spin-orbit coupling and strong anomalous Hall effect in the Heusler compensated ferrimagnet $\mathrm{Ti}_{2} \mathrm{MnAl}$, Phys. Rev. B 97, 060406 (2018).

[54] J.-R. Soh, F. de Juan, M. G. Vergniory, N. B. M. Schröter, M. C. Rahn, D. Y. Yan, J. Jiang, M. Bristow, P. Reiss, J. N. Blandy, Y. F. Guo, Y. G. Shi, T. K. Kim, A. McCollam, S. H. Simon, Y. Chen, A. I. Coldea, and A. T. Boothroyd, Ideal Weyl semimetal induced by magnetic exchange, Phys. Rev. B 100, 201102 (2019)

[55] I. Belopolski, K. Manna, D. S. Sanchez, G. Chang, B. Ernst, J. Yin, S. S. Zhang, T. Cochran, N. Shumiya, H. Zheng, B. Singh, G. Bian, D. Multer, M. Litskevich, X. Zhou, S.-M. Huang, B. Wang, T.-R. Chang, S.-Y. Xu, A. Bansil, C. Felser, H. Lin, and M. Z. Hasan, Discovery of topological Weyl fermion lines and drumhead surface states in a room temperature magnet, Science 365, 1278 (2019)

[56] P. Li, J. Koo, W. Ning, J. Li, L. Miao, L. Min, Y. Zhu, Y. Wang, N. Alem, C.-X. Liu, Z. Mao, and B. Yan, Giant room temperature anomalous Hall effect and tunable topology in a ferromagnetic topological semimetal $\mathrm{Co}_{2} \mathrm{MnAl}$, Nature Communications 11, 3476 (2020)

[57] J. Hofmann and S. Das Sarma, Surface plasmon polaritons in topological Weyl semimetals, Phys. Rev. B 93, 241402 (2016)

[58] O. V. Kotov and Y. E. Lozovik, Giant tunable nonreciprocity of light in Weyl semimetals, Phys. Rev. B 98, 195446 (2018)

[59] T. Tamaya, T. Kato, K. Tsuchikawa, S. Konabe, and S. Kawabata, Surface plasmon polaritons in thin-film Weyl semimetals, Journal of Physics: Condensed Matter 31, 305001 (2019)

[60] S. O. Abdol, A. S. Vala, and B. Abdollahipour, Tunable surface plasmon polaritons in a Weyl semimetal waveguide, Journal of Physics: Condensed Matter 31, 335002 (2019).

[61] L. Zhu and S. Fan, Near-complete violation of detailed balance in thermal radiation, Phys. Rev. B 90, 220301 (2014)

[62] D. A. B. Miller, L. Zhu, and S. Fan, Universal modal radiation laws for all thermal emitters, Proceedings of the National Academy of Sciences 114, 4336 (2017).

[63] L. Zhu, Y. Guo, and S. Fan, Theory of many-body radiative heat transfer without the constraint of reciprocity, Phys. Rev. B 97,
$094302(2018)$

[64] B. Zhao, Y. Shi, J. Wang, Z. Zhao, N. Zhao, and S. Fan, Nearcomplete violation of Kirchhoff's law of thermal radiation with a 0.3T magnetic field, Opt. Lett. 44, 4203 (2019)

[65] C. Khandekar and Z. Jacob, Thermal spin photonics in the near-field of nonreciprocal media, New Journal of Physics 21, 103030 (2019)

[66] L. Fan, Y. Guo, G. T. Papadakis, B. Zhao, Z. Zhao, S. Buddhiraju, M. Orenstein, and S. Fan, Nonreciprocal radiative heat transfer between two planar bodies, Phys. Rev. B 101, 085407 (2020)

[67] Y. Zhang, C.-L. Zhou, H.-L. Yi, and H.-P. Tan, Radiative thermal diode mediated by nonreciprocal graphene plasmon waveguides, Phys. Rev. Applied 13, 034021 (2020)

[68] B. Zhao, C. Guo, C. A. C. Garcia, P. Narang, and S. Fan, Axionfield-enabled nonreciprocal thermal radiation in Weyl semimetals, Nano Letters 20, 1923 (2020)

[69] C. Guo, B. Zhao, D. Huang, and S. Fan, Radiative thermal router based on tunable magnetic Weyl semimetals, ACS Photonics 7, 3257 (2020)

[70] Y. Tsurimaki, X. Qian, S. Pajovic, F. Han, M. Li, and G. Chen, Large nonreciprocal absorption and emission of radiation in type-I Weyl semimetals with time reversal symmetry breaking, Phys. Rev. B 101, 165426 (2020)

[71] S. Pajovic, Y. Tsurimaki, X. Qian, and G. Chen, Intrinsic nonreciprocal reflection and violation of Kirchhoff's law of radiation in planar type-I magnetic Weyl semimetal surfaces, Phys. Rev. B 102, 165417 (2020)

[72] G. Xu, J. Sun, and H. Mao, Near-field radiative thermal modulation between Weyl semimetal slabs, Journal of Quantitative Spectroscopy and Radiative Transfer 253, 107173 (2020)

[73] X. Wan, A. M. Turner, A. Vishwanath, and S. Y. Savrasov, Topological semimetal and Fermi-arc surface states in the electronic structure of pyrochlore iridates, Phys. Rev. B 83, 205101 (2011)

[74] N. P. Armitage, E. J. Mele, and A. Vishwanath, Weyl and Dirac semimetals in three-dimensional solids, Rev. Mod. Phys. 90, 015001 (2018)

[75] A. A. Zyuzin and A. A. Burkov, Topological response in Weyl semimetals and the chiral anomaly, Phys. Rev. B 86, 115133 (2012)

[76] O. V. Kotov and Y. E. Lozovik, Dielectric response and novel electromagnetic modes in three-dimensional Dirac semimetal films, Phys. Rev. B 93, 235417 (2016) 


\section{Supporting Information}

When the incidence plane is at azimuthal angle $\phi$ with respect to the $x$-axis, the dielectric tensor becomes

$$
\overline{\bar{\epsilon}}^{\prime}=\mathcal{R} \overline{\bar{\epsilon}} \mathcal{R}^{T}=\left[\begin{array}{lll}
\epsilon_{x x} & \epsilon_{x y} & \epsilon_{x z} \\
\epsilon_{y x} & \epsilon_{y y} & \epsilon_{y z} \\
\epsilon_{z x} & \epsilon_{z y} & \epsilon_{z z}
\end{array}\right]
$$

with the rotation matrix

$$
\mathcal{R}=\left[\begin{array}{ccc}
\cos (\phi+\theta) & -\sin (\phi+\theta) & 0 \\
\sin (\phi+\theta) & \cos (\phi+\theta) & 0 \\
0 & 0 & 1
\end{array}\right]
$$

For the lower WSM, we put $\theta=0$. We first focus on the interface between the lower Weyl semimetal (WSM) and air. The general form of the electric and magnetic fields inside the WSM can be written as

$$
\mathbf{E}=\left(\mathcal{E}_{x}, \mathcal{E}_{y}, \mathcal{E}_{z}\right) e^{i q x-i \omega t}, \quad \mathbf{H}=\left(\mathcal{H}_{x}, \mathcal{H}_{y}, \mathcal{H}_{z}\right) e^{i q x-i \omega t},
$$

where the superscript ' in the space variables $x^{\prime}, y^{\prime}$ and $z^{\prime}$ is dropped for simplicity. From Maxwell equations, we get the differential equation

$$
d M / d z=i K M
$$

where $M=\left[\mathcal{E}_{x}, \mathcal{E}_{y}, \alpha \mathcal{H}_{x}, \alpha \mathcal{H}_{y}\right]^{T}$ with $\alpha=\sqrt{\mu_{0} / \epsilon_{0}}$ and

$$
K=\left[\begin{array}{cccc}
-q \epsilon_{z x} / \epsilon_{z z} & -q \epsilon_{z y} / \epsilon_{z z} & 0 & k_{0}-q^{2} /\left(k_{0} \epsilon_{z z}\right) \\
0 & 0 & -k_{0} & 0 \\
k_{0}\left(-\epsilon_{y x}+\epsilon_{y z} \epsilon_{z x} / \epsilon_{z z}\right) & k_{0}\left(-\epsilon_{y y}+\epsilon_{y z} \epsilon_{z y} / \epsilon_{z z}\right)+q^{2} / k_{0} & 0 & q \epsilon_{y z} / \epsilon_{z z} \\
k_{0}\left(\epsilon_{x x}-\epsilon_{x z} \epsilon_{z x} / \epsilon_{z z}\right) & k_{0}\left(\epsilon_{x y}-\epsilon_{x z} \epsilon_{z y} / \epsilon_{z z}\right) & 0 & -q \epsilon_{x z} / \epsilon_{z z}
\end{array}\right]
$$

This differential equation has the solution

$$
\begin{aligned}
& {\left[\mathcal{E}_{x}(z), \mathcal{E}_{y}(z), \alpha \mathcal{H}_{x}(z), \alpha \mathcal{H}_{y}(z)\right] } \\
= & \sum_{m=1}^{2} c_{m}\left[u_{1, m}, u_{2, m}, u_{3, m}, u_{4, m}\right] e^{i k_{m} z}
\end{aligned}
$$

where $k_{m}$ and $u_{i, m}$ are, respectively, the eigenvalue and eigenvector of matrix $K$. Since $K$ is a four-by-four matrix, there are four eigenvalues: two of them satisfy $\operatorname{Im}\left(k_{m}\right)<0$ and the other two $\operatorname{Im}\left(k_{m}\right)>0$. We take $k_{m}$ with $\operatorname{Im}\left(k_{m}\right)<$ 0 , of which the subscripts are denoted as $m=1,2$, to ensure that the electromagnetic fields vanish at $z \rightarrow-\infty$.

The incoming electric and magnetic fields in air can be, respectively, written as

$$
\begin{aligned}
\mathbf{E}_{\text {in }} & =\left[e_{\text {in }}^{s} \hat{y}+e_{\text {in }}^{p}\left(\beta_{0} \hat{x}-q \hat{z}\right) / k_{0}\right] e^{i q x+i \beta_{0} z-i \omega t}, \\
\alpha \mathbf{H}_{\text {in }} & =\left[e_{\text {in }}^{p} \hat{y}-e_{\text {in }}^{s}\left(\beta_{0} \hat{x}-q \hat{z}\right) / k_{0}\right] e^{i q x+i \beta_{0} z-i \omega t},
\end{aligned}
$$

where the superscripts $s$ and $p$ are used to denote the polarization states. The reflected fields are then expressed as

$$
\begin{aligned}
\mathbf{E}_{\mathrm{re}} & =\left[e_{\mathrm{re}}^{s} \hat{y}-e_{\mathrm{re}}^{p}\left(\beta_{0} \hat{x}+q \hat{z}\right) / k_{0}\right] e^{i q x-i \beta_{0} z-i \omega t}, \\
\alpha \mathbf{H}_{\mathrm{re}} & =\left[e_{\mathrm{re}}^{p} \hat{y}+e_{\mathrm{re}}^{s}\left(\beta_{0} \hat{x}+q \hat{z}\right) / k_{0}\right] e^{i q x-i \beta_{0} z-i \omega t} .
\end{aligned}
$$

At the interface of the air side with $z=0^{+}$, the in-plane components of the electric and magnetic fields are

$$
\begin{aligned}
\mathbf{E}_{\|} & =\left[\left(e_{\mathrm{in}}^{s}+e_{\mathrm{re}}^{s}\right) \hat{y}+\left(e_{\mathrm{in}}^{p}-e_{\mathrm{re}}^{p}\right) \beta_{0} / k_{0} \hat{x}\right] e^{i q x-i \omega t}, \\
\alpha \mathbf{H}_{\|} & =\left[\left(e_{\mathrm{in}}^{p}+e_{\mathrm{re}}^{p}\right) \hat{y}+\left(e_{\mathrm{re}}^{s}-e_{\mathrm{in}}^{s}\right) \beta_{0} / k_{0} \hat{x}\right] e^{i q x-i \omega t} .
\end{aligned}
$$

For the case of the $p$-polarized incoming field, that is, $e_{\mathrm{in}}^{s}=$
0 , the interface conditions give

$$
\begin{array}{r}
\left(e_{\mathrm{in}}^{p}-e_{\mathrm{re}}^{p}\right) \beta_{0} / k_{0}=c_{1} u_{1,1}+c_{2} u_{1,2}, \\
e_{\mathrm{re}}^{s}=c_{1} u_{2,1}+c_{2} u_{2,2}, \\
e_{\mathrm{re}}^{s} \beta_{0} / k_{0}=c_{1} u_{3,1}+c_{2} u_{3,2}, \\
e_{\mathrm{in}}^{p}+e_{\mathrm{re}}^{p}=c_{1} u_{4,1}+c_{2} u_{4,2} .
\end{array}
$$

From Eqs. 29, and 30, we have

$$
c_{2} / c_{1}=-\left(u_{2,1} \beta_{0}-u_{3,1} k_{0}\right) /\left(u_{2,2} \beta_{0}-u_{3,2} k_{0}\right) .
$$

The reflection coefficient $r^{p p}=e_{\mathrm{re}}^{p} / e_{\mathrm{in}}^{p}$ can be obtained from Eqs. (28) and (31) as

$$
r^{p p}=\frac{\left(u_{4,1} \beta_{0}-u_{1,1} k_{0}\right)+\left(u_{4,2} \beta_{0}-u_{1,2} k_{0}\right) c_{2} / c_{1}}{\left(u_{4,1} \beta_{0}+u_{1,1} k_{0}\right)+\left(u_{4,2} \beta_{0}+u_{1,2} k_{0}\right) c_{2} / c_{1}} .
$$

From Eqs. 28) and (30), we obtain $r^{s p}=e_{\mathrm{re}}^{s} / e_{\mathrm{in}}^{p}$ as

$$
r^{s p}=\left(1-r^{p p}\right) \frac{u_{3,1}+u_{3,2} c_{2} / c_{1}}{u_{1,1}+u_{1,2} c_{2} / c_{1}} .
$$

For the case of the $s$-polarized incoming field, that is, $e_{\text {in }}^{p}=$ 0 , the interface conditions give

$$
\begin{aligned}
-e_{\mathrm{re}}^{p} \beta_{0} / k_{0} & =d_{1} u_{1,1}+d_{2} u_{1,2}, \\
e_{\mathrm{in}}^{s}+e_{\mathrm{re}}^{s} & =d_{1} u_{2,1}+d_{2} u_{2,2}, \\
\left(e_{\mathrm{re}}^{s}-e_{\mathrm{in}}^{s}\right) \beta_{0} / k_{0} & =d_{1} u_{3,1}+d_{2} u_{3,2}, \\
e_{\mathrm{re}}^{p} & =d_{1} u_{4,1}+d_{2} u_{4,2} .
\end{aligned}
$$

Here, we have use $d_{1}$ and $d_{2}$ instead of $c_{1}$ and $c_{2}$ to distinguish from the case of the $p$-polarized incoming field. From Eqs. (35) and (38), we have

$$
d_{2} / d_{1}=-\left(u_{4,1} \beta_{0}+u_{1,1} k_{0}\right) /\left(u_{4,2} \beta_{0}+u_{1,2} k_{0}\right) .
$$


The reflection coefficient $r^{s s}=e_{\mathrm{re}}^{s} / e_{\mathrm{in}}^{s}$ can be obtained from Eqs. 36 and 37) as

$$
r^{s s}=\frac{\left(u_{2,1} \beta_{0}+u_{3,1} k_{0}\right)+\left(u_{2,2} \beta_{0}+u_{3,2} k_{0}\right) d_{2} / d_{1}}{\left(u_{2,1} \beta_{0}-u_{3,1} k_{0}\right)+\left(u_{2,2} \beta_{0}-u_{3,2} k_{0}\right) d_{2} / d_{1}} .
$$

From Eqs. 35 and 37, we obtain $r^{p s}=e_{\text {re }}^{p} / e_{\text {in }}^{s}$ as

$$
r^{p s}=\left(1-r^{s s}\right) \frac{u_{1,1}+u_{1,2} d_{2} / d_{1}}{u_{3,1}+u_{3,2} d_{2} / d_{1}} .
$$

The reflection coefficient matrix at the interface between air and WSM $n$ with $n=1,2$ is given by

$$
\mathbf{R}_{n}=\left[\begin{array}{ll}
r_{n}^{p p} & r_{n}^{p s} \\
r_{n}^{s p} & r_{n}^{s s}
\end{array}\right]
$$

For the particular case of $\phi=\theta=0$, Eq. 19 reduces to

$$
\frac{d}{d z}\left[\begin{array}{c}
\mathcal{E}_{x} \\
\alpha \mathcal{H}_{y}
\end{array}\right]=i K^{p}\left[\begin{array}{c}
\mathcal{E}_{x} \\
\alpha \mathcal{H}_{y}
\end{array}\right], \quad \frac{d}{d z}\left[\begin{array}{c}
\mathcal{E}_{y} \\
\alpha \mathcal{H}_{x}
\end{array}\right]=i K^{s}\left[\begin{array}{c}
\mathcal{E}_{y} \\
\alpha \mathcal{H}_{x}
\end{array}\right],
$$

with

$$
K^{p}=\left[\begin{array}{cc}
i q \epsilon_{a} / \epsilon_{d} & k_{0}-q^{2} /\left(k_{0} \epsilon_{d}\right) \\
k_{0}\left(\epsilon_{d}-\epsilon_{a}^{2} / \epsilon_{d}\right) & -i q \epsilon_{a} / \epsilon_{d}
\end{array}\right]
$$

and

$$
K^{s}=\left[\begin{array}{cc}
0 & -k_{0} \\
-k_{0} \epsilon_{d}+q^{2} / k_{0} & 0
\end{array}\right]
$$

One can find that $\beta_{1}$ under $\phi=0$ satisfies $\operatorname{det}\left(\beta_{1}-K^{p}\right)=0$ and the solution to $\operatorname{det}\left(\beta_{1}^{\prime}-K^{s}\right)=0$ is given by $\left(\beta_{1}^{\prime}\right)^{2}+q^{2}=$ $\epsilon_{d} k_{0}^{2}$ with $\operatorname{Im}\left(\beta_{1}^{\prime}\right)<0$. The reflection coefficients for the $p$ and $s$-polarized modes are, respectively, given by

$$
r^{p p}=\frac{\epsilon_{\mathrm{eff}} \beta_{0}-\left(\beta_{1}+i q \epsilon_{a} / \epsilon_{d}\right)}{\epsilon_{\mathrm{eff}} \beta_{0}+\left(\beta_{1}+i q \epsilon_{a} / \epsilon_{d}\right)}, \quad r^{s s}=\frac{\beta_{0}-\beta_{1}^{\prime}}{\beta_{0}+\beta_{1}^{\prime}},
$$

and the other reflection coefficients vanish. The photonic transmission coefficient in this particular case is expressed as

$$
\xi(\omega, q, \phi=0)=\frac{4\left[\operatorname{Im}\left(r^{p p}\right)\right]^{2} e^{-2\left|\beta_{0}\right| d}}{\mid 1-\left(r^{p p}\right)^{2} e^{-\left.2\left|\beta_{0}\right| d\right|^{2}}}+\left[r^{p p} \rightarrow r^{s s}\right] .
$$



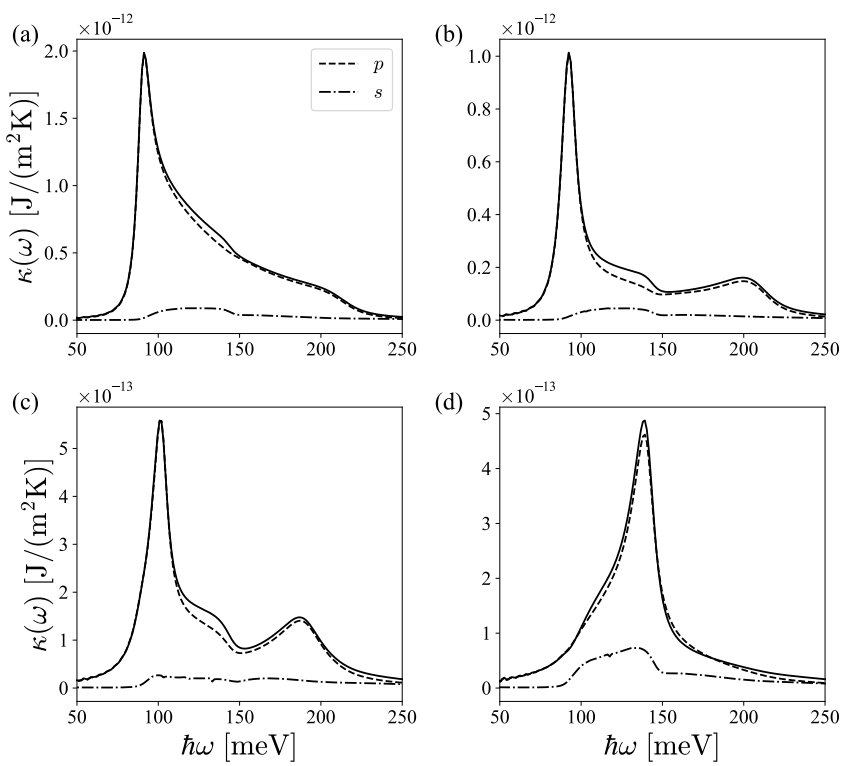

FIG. 5. Spectral function $\kappa(\omega)$ at twist angles (a) $\theta=0$, (b) $\theta=$ $\pi / 4$, (c) $\theta=\pi / 2$, and (d) $\theta=\pi$ with $d=100 \mathrm{~nm}$. The solid lines are obtained by considering the contributions from all the elements in the reflection coefficient matrix $\mathbf{R}_{n}$. The dashed (dash-dotted) lines give the contribution from the $p$-polarized ( $s$-polarized) mode by setting $r_{n}^{p s}=r_{n}^{s p}=r_{n}^{s s}=0\left(r_{n}^{p s}=r_{n}^{s p}=r_{n}^{p p}=0\right)$ in $\mathbf{R}_{n}$. It can be infered from the lines that the $p$-polarized mode dominates the near-field radiative heat transfer. 